\title{
Aberrant hypermethylation and reduced expression of disabled-2 promote the development of lung cancers
}

\author{
XUE-MEI XIE ${ }^{1,2}$, ZI-YIN ZHANG $^{3}$, LIAN-HE YANG $^{1}$, DA-LEI YANG ${ }^{4}$, NA TANG $^{1}$, \\ HUAN-YU ZHAO ${ }^{1}$, HONG-TAO XU ${ }^{1}$, QING-CHANG LI $^{1}$ and EN-HUA WANG ${ }^{1}$ \\ ${ }^{1}$ Department of Pathology, The First Affiliated Hospital and College of Basic Medical Sciences \\ of China Medical University, Shenyang 110001; ${ }^{2}$ Department of Pathology, Chengdu Military General Hospital, \\ Chengdu 610083; ${ }^{3}$ Department of Neurosurgery, Meishan People's Hospital, Meishan, Sichuan 620010; ${ }^{4}$ Assisted \\ Reproduction Center, Shengjing Hospital of China Medical University, Shenyang 110004, P.R. China
}

Received July 3, 2013; Accepted August 9, 2013

DOI: 10.3892/ijo.2013.2084

\begin{abstract}
Disabled-2 (Dab2) is considered a tumor suppressor and is downregulated in cancers. We examined the promoter methylation status and expression levels of Dab2, and investigated their roles in the development of lung cancers. Methylation-specific PCR was employed to analyze the methylation status of Dab2 in 100 lung cancer tissues. The cytoplasmic and nuclear expression of the Dab2 protein was determined using western blot analysis. Demethylation treatment using 5-Aza-2-deoxycytidine (5-Aza-dC) was performed in three lung cancer cell lines. Dab2 expression was upregulated by $D a b 2$ transfection or interrupted by Dab2 siRNA in lung cancer cells. Proliferative and invasive ability tests were performed with 3-(4,5-dimethylthiazol2-yl)-2,5-diphenyltetrazolium bromide (MTS) and a Matrigel invasion assay, respectively. The methylation rate of $D a b 2$ was significantly higher in lung cancer tissues compared to normal lung tissues. Dab2 methylation correlated with the reduced nuclear and cytoplasmic expression of Dab2, as well as the TNM stage and lymphatic metastasis of lung cancers. Treatment with 5-Aza-dC was able to eliminate the hypermethylation of Dab2, enhance Dab2 expression, and inhibit $\beta$-catenin expression, and the proliferative and invasive ability of lung cancer cells. Upregulation of Dab2 expression reduced $\beta$-catenin expression and proliferation and invasiveness of lung cancer cells. However, interruption of Dab2 expression induced the opposite results. Dab2 methylation is common in lung cancers, and is one of the most important factors responsible for the reduced expression of Dab2. Aberrant
\end{abstract}

Correspondence to: Professor Hong-Tao Xu, Department of Pathology, The First Affiliated Hospital and College of Basic Medical Sciences of China Medical University, Shenyang 110001, P.R. China

E-mail: htxu@mail.cmu.edu.cn

Key words: disabled-2, methylation, lung cancer, proliferation, invasion hypermethylation and reduced expression of Dab2 promote the development of lung cancers.

\section{Introduction}

The abnormal activation of Wnt/Wingless signaling pathway has been confirmed to be related to tumorigenesis in many tumor types $(1,2)$. When the Wnt signal is weak, $\beta$-catenin is incorporated in a destruction complex that contains glycogen synthase kinase 3 (GSK3), adenomatous polyposis coli (APC), axin, and casein kinase I (CKI), which results in phosphorylation of $\beta$-catenin; phosphorylated $\beta$-catenin is then degraded by a ubiquitin-mediated proteasomal pathway (3). However, in human cancers, the dissociation of $\beta$-catenin from this destruction complex results in the accumulation of $\beta$-catenin in the cytoplasm and nucleus, then activates the target genes of Wnt pathway, such as cyclin D1 and c-myc $(1,2,4)$. Axin, a key member of the destruction complex, can be recruited to the plasma membrane by low-density lipoprotein receptor-related protein (LRP) 5/6 co-receptors, which is facilitated by dishevelled. This translocation will induce axin dephosphorylation by protein phosphatase 1 (PP1), resulting in its degradation (5-7).

Disabled-2 (Dab2) is a member of the Mammalia/ Drosophila disabled gene family (8), and contains 2 isoforms (p67 and p96) (9). It is a widely expressed endocytic adapter protein, and a regulator of some receptor-mediated signaling pathways $(2,10-12)$. Dab2 can stabilize axin by preventing its interaction with LRP5/6 co-receptors and PP1, or promote LRP6 internalization through clathrin, and serves as an inhibitor of the Wnt pathway $(5,13,14)$. Abnormal expression of Dab2 plays an important role in cell differentiation and in the origin and development of cancers (15-18). However, the expression level of Dab2 and its significance for the development of lung cancers remains unclear.

In this study, we examined the relationship among Dab2 promoter methylation status, reduced expression of Dab2, and clinicopathological characteristics in lung cancers. In addition, we regulated the methylation status or expression level of Dab2 in lung cancer cells in order to investigate the mechanisms of Dab2 in the regulation of proliferation and invasiveness. 
Table I. Correlations between Dab2 methylation and lung cancer clinicopathological factors.

\begin{tabular}{|c|c|c|c|c|c|}
\hline \multirow{2}{*}{$\begin{array}{l}\text { Clinicopathological } \\
\text { factors }\end{array}$} & \multirow[b]{2}{*}{$\mathrm{n}$} & \multicolumn{2}{|c|}{ Methylation of Dab2 } & \multirow[b]{2}{*}{$\chi^{2}$ value } & \multirow[b]{2}{*}{ P-value } \\
\hline & & Positive & Negative & & \\
\hline Gender & & & & 0.002 & 0.962 \\
\hline Male & 58 & 54 & 4 & & \\
\hline Female & 42 & 39 & 3 & & \\
\hline Age & & & & 0.227 & 0.248 \\
\hline$<60$ & 64 & 61 & 3 & & \\
\hline$\geq 60$ & 36 & 32 & 4 & & \\
\hline Histologic type & & & & 7.658 & 0.022 \\
\hline Adenocarcinoma & 53 & 52 & 1 & & \\
\hline Squamous cell carcinoma & 38 & 32 & 6 & & \\
\hline Small cell lung cancer & 9 & 9 & 0 & & \\
\hline Differentiation & & & & 9.703 & 0.009 \\
\hline Well & 24 & 19 & 5 & & \\
\hline Moderate & 50 & 48 & 2 & & \\
\hline Poor & 26 & 26 & 0 & & \\
\hline Lymphatic metastasis & & & & 7.527 & 0.019 \\
\hline Yes & 50 & 50 & 0 & & \\
\hline No & 50 & 43 & 7 & & \\
\hline TNM stage & & & & 9.933 & 0.007 \\
\hline I & 51 & 44 & 7 & & \\
\hline II & 32 & 32 & 0 & & \\
\hline III & 17 & 17 & 0 & & \\
\hline
\end{tabular}

\section{Materials and methods}

Patients and specimens. A total of 100 paired fresh samples of primary lung cancer and corresponding normal lung tissues were selected randomly from patients diagnosed with lung cancer who underwent surgery at The First Affiliated Hospital of China Medical University between 2010 and 2012. The age of patients ranged from 37 to 82 years, and the mean age was 60 years (58 men and 42 women). The details of tumors were listed in Table I. Tumors were classified according to the system of the World Health Organization (2004), and the TNM classification scheme of the International Union Against Cancer. The study was conducted according to the regulations of the institutional review boards at China Medical University. Fresh tissue samples were stored at $-70^{\circ} \mathrm{C}$ immediately following resection.

Cell lines. A549, H157, H1299 and H460 cell lines were obtained from the ATCC (Manassas, VA, USA). LTEPa-2 (hereafter referred to as LTE), SPC and LK2 cell lines were obtained from the Cell Bank of the Chinese Academy (Shanghai, China). The BE1 cell line was kindly provided by Professor J. Zheng (Medical College of Beijing University, China) (19-21). A549 and LK2 cells were grown in Dulbecco's modified Eagle's medium (DMEM); other cells were cultured in RPMI-1640, supplemented with $10 \%$ fetal bovine serum (FBS) at $37^{\circ} \mathrm{C}$ in a $5 \% \mathrm{CO}_{2}$ humidified atmosphere.

DNA extraction and methylation-specific PCR (MSP) analysis. Genomic DNA was isolated from tissue samples or cells with a tissue/cell DNA extraction reagent kit (Bioteke, Beijing, China) according to the manufacturer's protocol. Bisulfite conversion of DNA was performed with the EZ DNA Methylation kit (Zymo Research, Beijing, China) according to the manufacturer's instructions. The primers of the nested PCR were as follows: forward, AAAGGTAGTTTTTTGT TTAAAGGG; reverse, TAAACTTAATAACTCCCCCTCA (product length: $367 \mathrm{bp}$ ). First, bisulfite-treated DNA was amplified for 30 cycles: $95^{\circ} \mathrm{C}$ for $5 \mathrm{~min}$, followed by cycling at $95^{\circ} \mathrm{C}$ for $30 \mathrm{sec}, 52^{\circ} \mathrm{C}$ for $30 \mathrm{sec}$, and $72^{\circ} \mathrm{C}$ for $45 \mathrm{sec}$, with a final extension step at $72^{\circ} \mathrm{C}$ for $10 \mathrm{~min}$. Next, the nested PCR products were diluted 100 times, and amplified for 45 cycles with MSP primers: $95^{\circ} \mathrm{C}$ for $5 \mathrm{~min}$, followed by cycling at $95^{\circ} \mathrm{C}$ for $30 \mathrm{sec}, 60^{\circ} \mathrm{C}$ for $30 \mathrm{sec}$, and $72^{\circ} \mathrm{C}$ for $30 \mathrm{sec}$, with a final extension step at $72^{\circ} \mathrm{C}$ for $10 \mathrm{~min}$. The primers of MSP were as follows: methylated forward, GGATTTGTGAAACGA AGTTC; methylated reverse, CACCAACTAAAAACGATCG (product length, 168 bp); un-methylated forward, GGATTTG TGAAATGAAGTTT; un-methylated reverse, CACCAACTA AAAACAATCA (product length, 168 bp). Finally, the MSP products were electrophoresized on $2 \%$ agarose gels containing ethidium bromide and analyzed using a Bio-Imaging system (UVP, Upland, CA, USA).

Protein extraction and western blot analysis. We randomly selected 50 paired lung cancer and corresponding normal lung tissues, in which methylation status had been examined previously, and extracted nuclear and cytoplasmic proteins separately using the Nuclear and Cytoplasmic Protein Extraction kit 
(Bioteke) according to the manufacturer's protocol. The quantified proteins were separated by electrophoresis on $10 \%$ SDS-PAGE and transferred onto polyvinylidene difluoride membranes. The membranes were subsequently blocked with $5 \%$ skim-milk for $2 \mathrm{~h}$ and incubated overnight at $4^{\circ} \mathrm{C}$ with anti-Dab2 (1:500, Santa Cruz Biotechnology Inc., Santa Cruz, CA, USA), anti- $\beta$-catenin (1:500, BD Transduction Laboratories, KY, USA), anti- $\beta$-actin (1:500, BD Transduction Laboratories), and anti-histone 3.1 (1:400, Signalway Antibody, College Park, MD, USA) antibodies. The membranes were then incubated with appropriate secondary antibodies at $37^{\circ} \mathrm{C}$ for $2 \mathrm{~h}$. The protein bands were detected using an enhanced chemiluminescence system (ECL Plus, Bio-Rad Biosciences, Hercules, CA, USA). The relative expression quantity was scored as the ratio of $\beta$-catenin or Dab2 protein intensity to $\beta$-actin or histone $\mathrm{H} 3.1$ staining intensity.

Demethylation assay. We performed a demethylation treatment in A549, LTE and H1299 cells, which showed complete methylation of Dab2 in the MSP examination. Cells were seeded in 6-well plates and allowed to confluence for $24 \mathrm{~h}$, then treated with 5-Aza-2-deoxycytidine (5-Aza-dC) at a concentration of $5 \mu \mathrm{M}$ for $72 \mathrm{~h}$. The medium was changed every day. Cells cultured in the routine medium without 5-Aza-dC served as negative controls.

Dab2 gene transfection and siRNA knockdown assay. The Dab2 expression vector pRK5-Dab2 was kindly provided by Professor P.H. Howe (The Lerner Research Institute, Cleveland Clinic Foundation, Cleveland, OH, USA) $(5,12)$. The A549, LTE and H1299 cells were transfected with pRK5Dab2 using Lipofectamine 2000 (Invitrogen, Carlsbad, CA, USA) according to the manufacturer's instructions. The cells transfected with empty vectors and un-transfected cells served as negative controls. Dab2 siRNA, control siRNA and siRNA reagent system were purchased from Santa Cruz Biotechnology Inc. The siRNA interference of Dab2 was performed according to the manufacturer's instructions.

Cell proliferation analysis. The A549 cells transfected with Dab2, interrupted with Dab2 siRNA, or treated with 5-Aza-dC, along with control cells, were grown in 96-well plates separately at a density of $2.0 \times 10^{5}$ cells $/ \mathrm{ml}$. Every $24 \mathrm{~h}$, adherent cells were harvested and analyzed using The CellTiter 96 Aqueous One Solution cell proliferation assay [3-(4,5-Dimethylthiazol2-yl)-2,5-Diphenyltetrazolium Bromide (MTS)] (Promega, Madison, WI, USA). The absorbance, which is directly proportional to the number of living cells in culture, was measured at $490 \mathrm{~nm}$ using a microplate reader.

Cell invasion assay. Cell invasive ability was examined using a 24-well Transwell with $8-\mu \mathrm{m}$ pore polycarbonate membrane inserts (Corning Inc., Corning, NY, USA). The A549 cells of each experimental group and corresponding control groups were seeded on the upper chamber of an insert coated with Matrigel (Sigma-Aldrich, Saint Louis, MO, USA) at a density of $5 \times 10^{5}$ cells/well in serum-free DMEM medium. The DMEM medium with $10 \%$ FBS was added to the lower chamber (600 $\mu \mathrm{l} /$ well). After 30 -h incubation, the cells remaining on the upper membrane were removed with PBS and cotton wool, whereas cells that had invaded through the membrane were
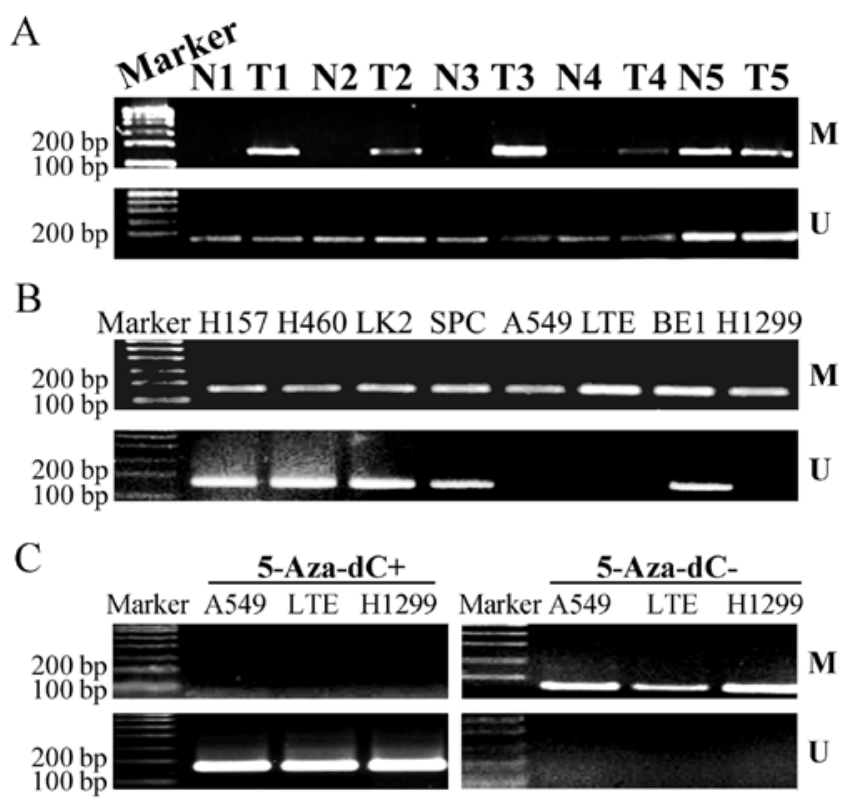

Figure 1. Promoter methylation of Dab2 in lung cancers and normal lung tissues. (A) The methylation rate of Dab2 in lung cancers was significantly higher than that in corresponding normal lung tissues. T, lung tumor tissues; N, normal lung tissues; M, methylated; U, unmethylated. (B) The methylation status of Dab2 in A549, LTE, H1299, H157, H460, LK2, SPC and BE1 lung cancer cells. (C) Treatment with 5-Aza-dC eliminated the methylation status of Dab2 in A549, LTE and H1299 cells.

fixed with paraformaldehyde and stained with hematoxylin. The cells were then viewed and counted using an IX71 inverted microscope (Olympus, Tokyo, Japan).

Statistical analysis. The paired sample t-tests was performed to analyze the cytoplasmic and nucleic expression level of Dab2 in lung cancers and the corresponding normal lung tissues. The independent $\mathrm{t}$-tests was used to evaluate the expression level of Dab2 in lung cancers between promotor methylated and unmethylated of Dab2 gene. The Pearson's $\chi^{2}$ test, or likelihood ratio test, was used to determine relationships between $D a b 2$ promoter methylation and clinicopathological characteristics of lung cancers or absent expression of p96-Dab2. The Spearman's correlation test was used to examine the correlations between protein expression levels and Dab2 methylation. Experiments of lung cancer cells were independently repeated 3 times. P-values $<0.05$ were considered statistically significant.

\section{Results}

Hypermethylation of Dab2 is common in lung cancers and correlates with clinicopathological parameters. In 100 lung cancer tissues, 58 cases $(58.0 \%)$ showed complete methylation, and 35 cases (35.0\%) showed incomplete methylation. However, in corresponding normal lung tissues, no case showed complete methylation, and 35 cases (35.0\%) showed incomplete methylation (Fig. 1A). So, the methylation rate of Dab2 in lung cancers (93.0\%) was significantly higher than that in corresponding normal lung tissues $(35 \%)(\mathrm{P}<0.001)$. More importantly, promoter methylation of Dab2 was correlated with differentiation $(\mathrm{P}=0.009)$, lymphatic metastasis 
A

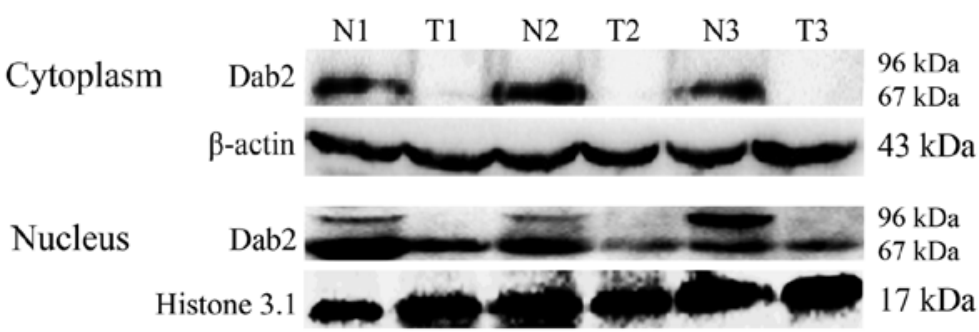

B

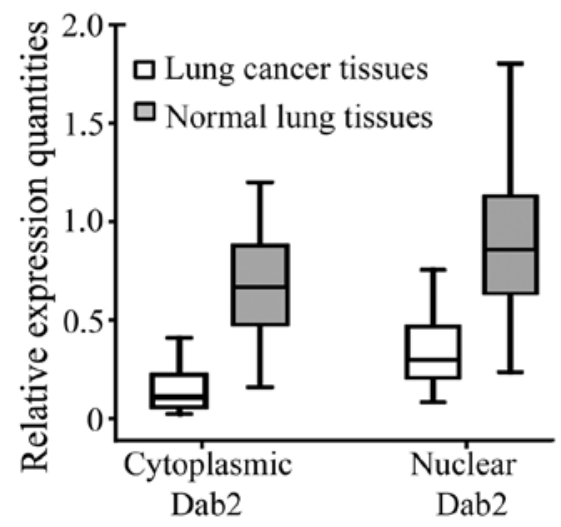

C

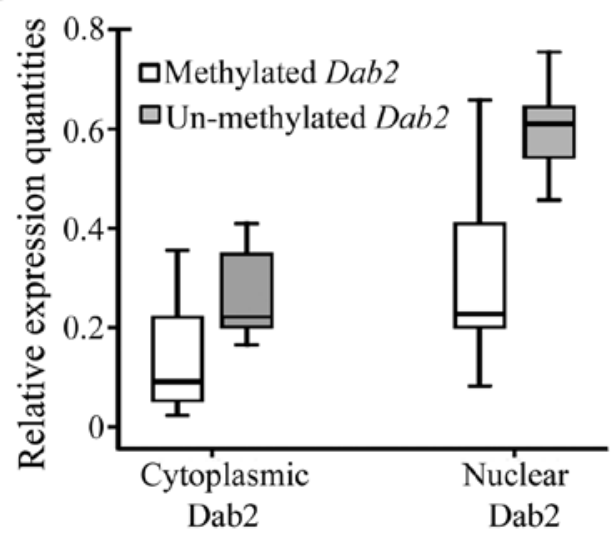

Figure 2. Nuclear and cytoplasmic expression of Dab2 in lung cancers and corresponding normal lung tissues. (A) The expression of p67-Dab2 was reduced in both the cytoplasm and the nucleus of lung cancer tissues compared to that in corresponding normal lung tissues. P96-Dab2 was expressed only in the nucleus of normal lung tissues, but lost in lung cancer tissues. $\beta$-actin and histone 3.1 served as internal controls in the cytoplasm and nucleus, respectively. T1, T2 and T3: lung tumor tissues; N1, N2 and N3: corresponding normal lung tissues. (B) The relative expression quantity of Dab2 in lung cancers (cytoplasmic expression, $0.151 \pm 0.109$; nuclear expression, $0.337 \pm 0.181$ ) was significantly less than that in corresponding normal lung tissues (cytoplasmic expression, $0.696 \pm 0.337$; nuclear expression, $0.901 \pm 0.384)(\mathrm{P}<0.001)$. (C) The relative expression quantity of Dab2 in lung cancers with Dab2 promoter methylation (cytoplasmic expression, $0.136 \pm 0.103$; nuclear expression, $0.301 \pm 0.158$ ) was much less than that in corresponding normal lung tissues (cytoplasmic expression, $0.261 \pm 0.095$; nuclear expression, $0.603 \pm 0.101)(\mathrm{P}<0.001)$.

$(\mathrm{P}=0.019), \mathrm{TNM}$ stage $(\mathrm{P}=0.007)$, and histological type $(\mathrm{P}=0.022)$, but not correlated with gender $(\mathrm{P}=0.962)$ or age $(\mathrm{P}=0.248)$ of the patients (Table I).

Dab2 is localized both in the cytoplasm and nucleus, and the reduced expression of Dab2 correlates with the promoter methylation of Dab2 gene. After examination of the methylation status, we selected 50 paired cases, to detect the cytoplasm and nucleus expression of Dab2. The expression of p67-Dab2 was observed both in the cytoplasm and nucleus of lung cancer and normal lung tissues. However, p96-Dab2 was expressed only in the nuclei of 31 cases $(31 / 50,62.0 \%)$ of normal lung tissues, and was lost in lung cancer tissues (Fig. 2A). The cytoplasmic or nuclear expression of Dab2 in lung cancers was significantly lower than that in normal lung tissues (for cytoplasmic expression: $0.151 \pm 0.109$ versus $0.696 \pm 0.337, \mathrm{t}=-10.836, \mathrm{P}<0.001$; for nuclear expression: $0.337 \pm 0.181$ versus $0.901 \pm 0.384 ; \mathrm{t}=-10.726, \mathrm{P}<0.001$ ) (Fig. 2B).

Moreover, cytoplasmic expression levels of Dab2 in lung cancer cases with Dab2 promoter methylation $(0.136 \pm 0.103)$ were significantly lower than that in lung cancer cases without Dab2 promoter methylation $(0.261 \pm 0.095$; $\mathrm{t}=-2.992$, $\mathrm{P}=0.021)$. The nuclear expression of Dab2 also showed similar results $(0.301 \pm 0.158$ versus $0.603 \pm 0.101 ; \mathrm{t}=-4.532, \mathrm{P}<0.001)$ (Fig. 2C). The Spearman's correlation tests confirmed that
Dab2 promoter methylation was negatively correlated with Dab2 expression levels in the cytoplasm (correlation coefficient, $-0.258, \mathrm{P}=0.009$ ) and in the nucleus (correlation coefficient, $-0.298, \mathrm{P}=0.003$ ) in lung cancer tissues. The loss of p96-Dab2 in corresponding normal lung tissues also correlated with the promoter methylation status of Dab2 $\left(\chi^{2}=12.063, \mathrm{P}=0.001\right)$. However, Dab2 expression levels did not correlate with clinicopathological parameters of patients (data not shown).

Treatment with 5-Aza-dC enhances the expression of Dab2, and inhibits the expression of $\beta$-catenin and the proliferative and invasive abilities of lung cancer cells. The promoter of Dab2 was methylated in all lung cancer cell lines used in this study. Complete methylation was observed in A549, LTE and H1299 cells, whereas incomplete methylation was observed in BE1, H460, SPC, H157 and LK2 cells (Fig. 1B). The completely methylated cells were then treated with 5 -Aza-dC for $72 \mathrm{~h}$ separately. The promoter methylation of Dab2 was successfully eliminated (Fig. 1C), and expression levels of Dab2 were increased significantly $(\mathrm{P}<0.05)$, whereas $\beta$-catenin were significantly reduced in lung cancer cells $(\mathrm{P}<0.05)$ (Fig. 3A). Furthermore, the invasive cell number of 5-Aza-dC-treated A549 cells $(9 \pm 2)$ was lower than that of untreated A549 cells $(20 \pm 4)(\mathrm{P}<0.05)$ (Fig. 3B and C). The growth rate of 5-Aza-dC-treated A549 cells was also reduced 
A

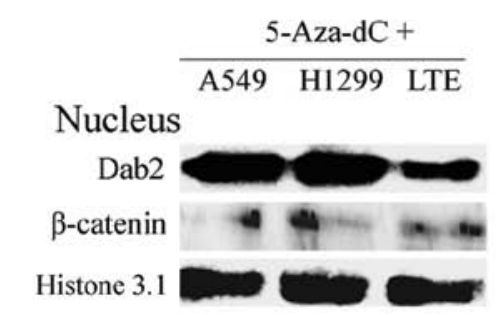

Cytoplasm

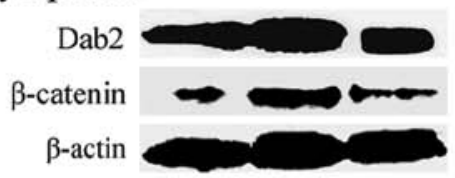

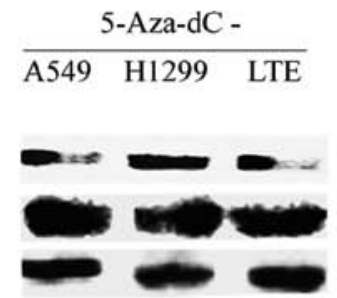

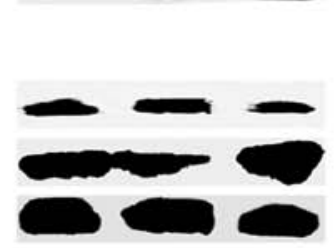

B

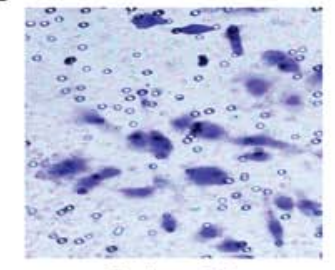

5-Aza-dC -

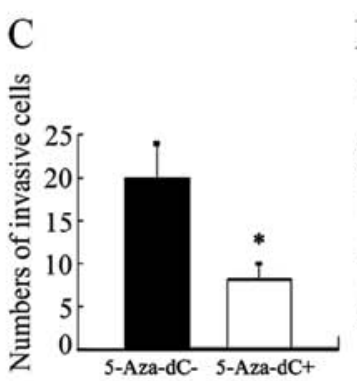

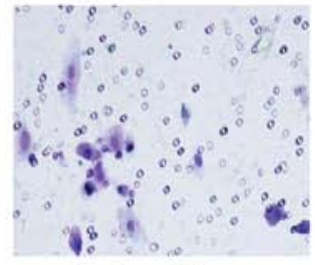

$5-\mathrm{Aza}-\mathrm{dC}+$

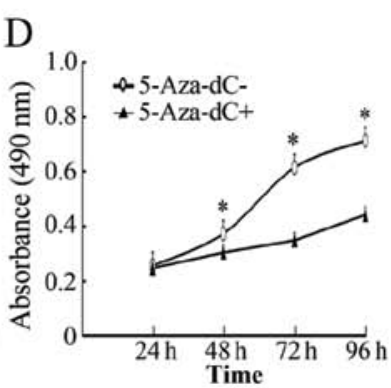

Figure 3. The expression of Dab2 and $\beta$-catenin and the proliferative and invasive ability of lung cancer cells after treatment with 5-Aza-dC. (A) Compared with untreated control cells (5-Aza-dC-), the nuclear and cytoplasmic expression of Dab2 was increased, and the nuclear and cytoplasmic expression of $\beta$-catenin was reduced in the A549, LTE and H1299 cells after 5-Aza-dC treatment. Histone 3.1 and $\beta$-actin served as internal controls in the nucleus and cytoplasm, respectively. (B) Representative microscope fields of filters under the Matrigel are shown from 5-Aza-dC-treated A549 cells and untreated control cells (5-Aza-dC-), respectively (original magnification, $\mathrm{x} 400$ ). (C) The number of invasive cells in 5-Aza-dC treated A549 cells was reduced relative to that of untreated control cells (5-Aza-dC-) (bar, SD; "P<0.05). (D) The growth curves indicated that the growth rate of 5-Aza-dC-treated A549 cells was reduced relative to that of untreated A549 cells (5-Aza-dC-) (bar, SD; $\left.{ }^{*} \mathrm{P}<0.05\right)$.
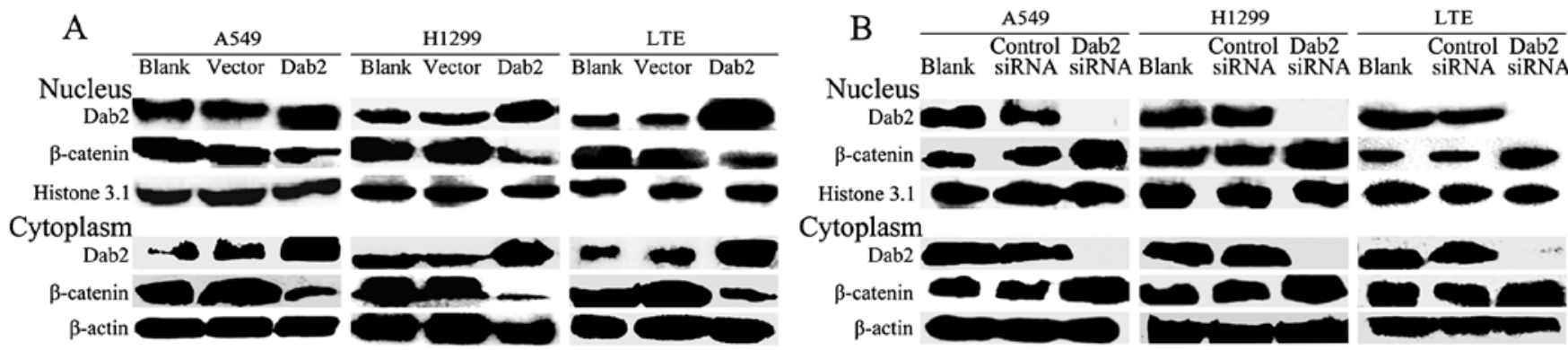

Figure 4. Nuclear and cytoplasmic expression of Dab2 and $\beta$-catenin in A549, H1299 and LTE cells with Dab2 transfection or Dab2 siRNA interference. (A) Both the nuclear and cytoplasmic expression of Dab2 in A549, LTE and H1299 cells with Dab2 gene transfection was significantly enhanced relative to the vector control cells or blank control cells. The expression, both nuclear and cytoplasmic, of $\beta$-catenin was markedly reduced. (B) The expression of Dab2 was absent or weak in A549, LTE and H1299 cells with Dab2 siRNA interference relative to the control siRNA or blank cells, whereas, both the nuclear and cytoplasmic expression of $\beta$-catenin was increased in A549, LTE and H1299 cells. Histone 3.1 and $\beta$-actin served as internal controls in the nucleus and in the cytoplasm, respectively.

relative to that of untreated A549 cells at the second, third, and fourth days of detection $(\mathrm{P}<0.05)$ (Fig. 3D).

Dab2 overexpression reduces the expression of $\beta$-catenin and inhibits the proliferative and invasive ability of lung cancer cells. Both the nuclear and cytoplasmic expression of Dab2 was significantly enhanced after Dab2 gene transfection in A549, LTE and H1299 cells $(\mathrm{P}<0.05)$. Whereas, the expression of $\beta$-catenin was reduced $(\mathrm{P}<0.05)$ (Fig. 4A). The invasive cell number of A549 cells after Dab2 gene transfection $(5 \pm 2)$ was reduced relative to the vector control A549 $(23 \pm 4)$ and untransfected A549 cells $(21 \pm 4)(\mathrm{P}<0.05)$ (Fig. 5A and B). The growth rate of Dab2-transfected A549 cells was also reduced relative to that of vector control A549 and untransfected A549 cells at the second, third, and fourth days of detection $(\mathrm{P}<0.05)$ (Fig. 5C).

Downregulation of the expression of Dab2 promotes the accumulation of $\beta$-catenin and enhances proliferation and invasiveness of lung cancer cells. After interference with Dab2 siRNA, both the nuclear and cytoplasmic expression of Dab2 was weak or absent in A549, LTE and H1299 cells, respectively $(\mathrm{P}<0.05)$. The expression of $\beta$-catenin was increased $(\mathrm{P}<0.05)$ (Fig. 4B). The invasive cell number of A549 cells with Dab2 siRNA interference $(60 \pm 5)$ was increased compared to the A549 cells treated with control siRNA (20 \pm 3$)$ and untreated 
A

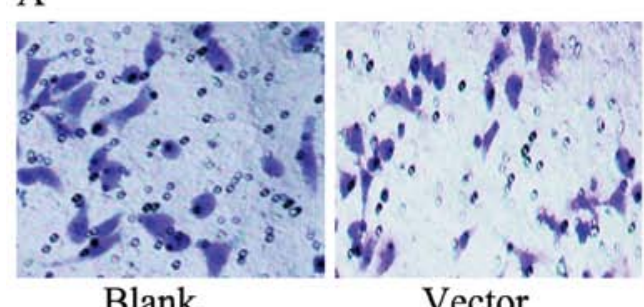

Blank

D

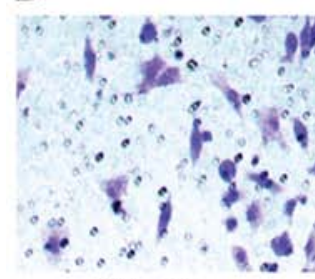

Blank
Vector

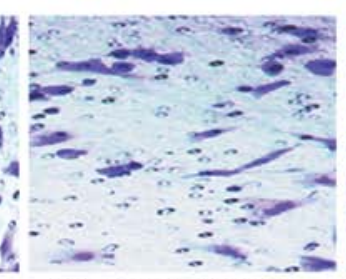

Control siRNA

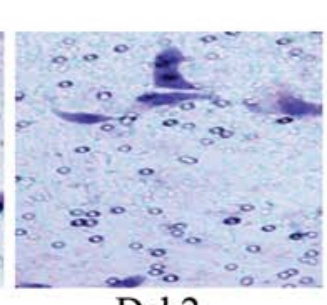

Dab2

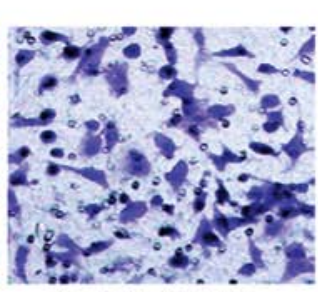

Dab2 siRNA
B

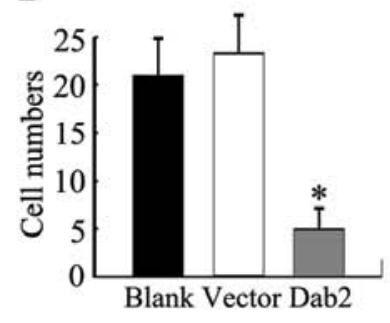

E

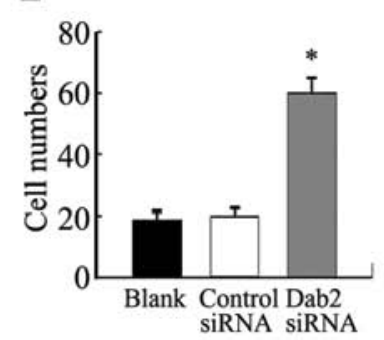

C
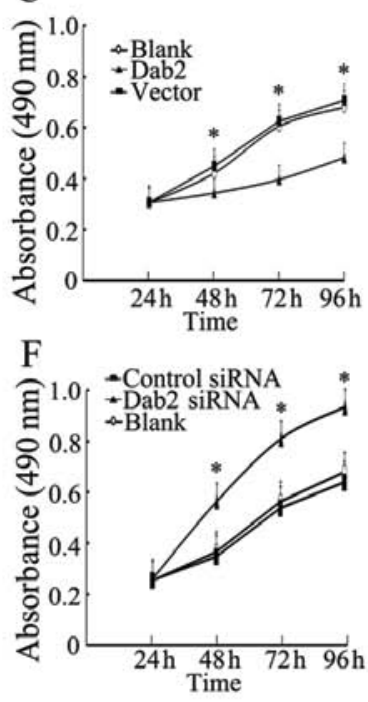

Figure 5. Invasiveness and proliferation of A549 cells with Dab2 gene transfection or Dab2 siRNA interference. (A) Representative microscope fields of filters under the Matrigel are shown from Dab2-transfected A549 cells, vector control A549 cells, and blank A549 cells, respectively (original magnification, x400). (B) The number of invasive cells in Dab2-transfected A549 cells was reduced relative to that of vector control A549 and blank A549 cells (bar, SD; ${ }^{*}<0.05$ ). (C) The growth curves indicated that the growth rate of Dab2-transfected A549 cells was reduced relative to that of vector control A549 and blank A549 cells (bar, SD; ${ }^{*} \mathrm{P}<0.05$ ). (D) The representative microscope fields of filters under the Matrigel are shown from A549 cells with Dab2 siRNA interference, control siRNA A549 cells, and blank A549 cells, respectively (original magnification, x400). (E) The number of invasive cells in A549 cells with Dab2 siRNA interference was increased relative to the control siRNA A549 and blank A549 cells (bar, SD; $\left.{ }^{*} \mathrm{P}<0.05\right)$. (F) The growth curves indicated that the growth rate of A549 cells with Dab2 siRNA interference was increased relative to the control siRNA A549 and blank A549 cells (bar, SD; $\left.{ }^{*} \mathrm{P}<0.05\right)$.

A549 cells (19 \pm 4$)(\mathrm{P}<0.05)$ (Fig. 5D and $\mathrm{E})$. The growth rate of A549 cells with Dab2 siRNA interference was also increased relative to A549 cells treated with control siRNA and untreated A549 cells at the second, third, and fourth days of detection $(\mathrm{P}<0.05)$ (Fig. 5F).

\section{Discussion}

Dab2 has been shown to be a widely expressed endocytic adaptor protein (10), and participates in a variety of physiological processes such as cell mitosis (22), endothelial cell differentiation (23), development of the central nervous system (24), and in the regulation of the TGF- $\beta /$ Smad (12), and Wnt/ $\beta$-catenin signaling pathways (13). Reduced expression of Dab2 will result in the activation of Wnt pathway. Furthermore, loss of Dab2 expression may facilitate the establishment of an autocrine TGF $\beta$ signalling loop, and promote TGF $\beta$-stimulated epithelial-to-mesenchymal transition, and therefore increase the propensity for metastasis (25). Although downregulation of Dab2 has previously been demonstrated in other cancers $(15,17,18,26)$, concrete explanations for this observation have yet to be well addressed.

We, for the first time, showed that Dab2 was expressed in the cytoplasm and nucleus using immunofluorescence (27), and western blot analysis, and its expression was significantly reduced in lung cancers, especially the p96 isoforms. We further showed that Dab2 overexpression inhibited the accumulation of $\beta$-catenin by Dab2 gene transfection, which conclusively inhibited proliferation and invasiveness of lung cancer cells. However, downregulation of the expression of Dab2 by Dab2 siRNA induced opposite results. We confirmed that reduced expression of Dab2 could induce the abnormal activation of Wnt pathway and promote the development of lung cancers.

Our study demonstrated that the methylation of Dab2 is common in lung cancers, similar to the reports in other tumors (23,28-30). Furthermore, the methylation of Dab2 was correlated with the differentiation, lymphatic metastasis, and TNM stage of lung cancers. Importantly, we found that the methylation of $D a b 2$ was significantly correlated with reduced expression of the Dab2 protein in lung cancers. After treatment with 5-Aza-dC in A549, LTE and H1299 cells, which show complete methylation of the Dab2 promoter, we found the methylation of the Dab2 promoter was eliminated, and the expression of Dab2 was restored, which resulted in downregulation of $\beta$-catenin and the inhibition of the proliferative and invasive abilities of lung cancer cells. These results therefore demonstrated that the hypermethylation of Dab2 is a contributing factor in the reduced protein expression in lung cancers, and is also related to the development of lung cancers. So, the development of methods that could eliminate the methylation status of Dab2 or enhance the expression of Dab2 would offer potential therapeutic treatments for lung cancers.

In conclusion, the methylation of the gene $D a b 2$ is common in lung cancers, and is one of the most important factors responsible for the reduced expression of Dab2. Furthermore, aberrant hypermethylation and reduced expression of Dab2 promote the development of lung cancers.

\section{Acknowledgements}

This study was supported by the National Science Foundation of China (Grant No. 81372497 to H.-T. Xu) and the Program 
for Liaoning Excellent Talents in University (Grant No. LJQ2011085 to H.-T. Xu).

\section{References}

1. Lustig B and Behrens J: The Wnt signaling pathway and its role in tumor development. J Cancer Res Clin Oncol 129: 199-221, 2003.

2. Prunier C, Hocevar BA and Howe PH: Wnt signaling: physiology and pathology. Growth Factors 22: 141-150, 2004.

3. Kimelman D and Xu W: beta-catenin destruction complex: insights and questions from a structural perspective. Oncogene 25: 7482-7491, 2006.

4. MacDonald BT, Tamai $\mathrm{K}$ and $\mathrm{He} \mathrm{X}$ : Wnt/beta-catenin signaling: components, mechanisms, and diseases. Dev Cell 17: 9-26, 2009 .

5. Jiang Y,Luo W and Howe PH: Dab2 stabilizes Axin and attenuates Wnt/beta-catenin signaling by preventing protein phosphatase 1 (PP1)-Axin interactions. Oncogene 28: 2999-3007, 2009.

6. He X, Semenov M, Tamai K and Zeng X: LDL receptor-related proteins 5 and 6 in Wnt/beta-catenin signaling: arrows point the way. Development 131: 1663-1677, 2004.

7. Zeng X, Tamai K, Doble B, et al: A dual-kinase mechanism for Wnt co-receptor phosphorylation and activation. Nature 438 873-877, 2005.

8. Gertler FB, Bennett RL, Clark MJ and Hoffmann FM: Drosophila abl tyrosine kinase in embryonic CNS axons: a role in axonogenesis is revealed through dosage-sensitive interactions with disabled. Cell 58: 103-113, 1989.

9. Kim JA, Bae SH, Choi YJ, Kim KH and Park SS: Feed-back regulation of disabled-2 (Dab2) p96 isoform for GATA-4 during differentiation of F9 cells. Biochem Biophys Res Commun 421: 591-598, 2012

10. Fu L, Rab A, Tang LP, Rowe SM, Bebok Z and Collawn JF: Dab2 is a key regulator of endocytosis and post-endocytic trafficking of the cystic fibrosis transmembrane conductance regulator. Biochem J 441: 633-643, 2012.

11. Hung WS, Huang CL, Fan JT, Huang DY, Yeh CF, Cheng JC and Tseng CP: The endocytic adaptor protein Disabled-2 is required for cellular uptake of fibrinogen. Biochim Biophys Acta 1823: 1778-1788, 2012.

12. Hocevar BA, Smine A, Xu XX and Howe PH: The adaptor molecule Disabled-2 links the transforming growth factor beta receptors to the Smad pathway. EMBO J 20: 2789-2801, 2001.

13. Jiang Y, Prunier C and Howe PH: The inhibitory effects of Disabled-2 (Dab2) on Wnt signaling are mediated through Axin. Oncogene 27: 1865-1875, 2008.

14. Jiang Y, He X and Howe PH: Disabled-2 (Dab2) inhibits Wnt/beta-catenin signalling by binding LRP6 and promoting its internalization through clathrin. EMBO J 31: 2336-2349, 2012.

15. Karam JA, Shariat SF, Huang HY, et al: Decreased DOC-2/DAB2 expression in urothelial carcinoma of the bladder. Clin Cancer Res 13: 4400-4406, 2007.
16. Yang DH, Smith ER, Cohen C, et al: Molecular events associated with dysplastic morphologic transformation and initiation of ovarian tumorigenicity. Cancer 94: 2380-2392, 2002.

17. Kleeff J, Huang Y, Mok SC, Zimmermann A, Friess H and Büchler MW: Down-regulation of DOC-2 in colorectal cancer points to its role as a tumor suppressor in this malignancy. Dis Colon Rectum 45: 1242-1248, 2002.

18. Anupam K, Tusharkant C, Gupta SD and Ranju R: Loss of disabled-2 expression is an early event in esophageal squamous tumorigenesis. World J Gastroenterol 12: 6041-6045, 2006.

19. Chetrit D, Barzilay L, Horn G, Bielik T, Smorodinsky NI and Ehrlich M: Negative regulation of the endocytic adaptor disabled-2 (Dab2) in mitosis. J Biol Chem 286: 5392-5403, 2011.

20. Yang DH, Smith ER, Roland IH, et al: Disabled-2 is essential for endodermal cell positioning and structure formation during mouse embryogenesis. Dev Biol 251: 27-44, 2002.

21. Zhu W, Zheng J and Fang W: Isolation and characterization of human lung cancer cell subline with different metastatic potential. Zhonghua Bing Li Xue Za Zhi 24: 136-138, 1995 (In Chinese).

22. Liu CR, Ma CS, Ning JY, You JF, Liao SL and Zheng J: Differential thymosin beta 10 expression levels and actin filament organization in tumor cell lines with different metastatic potential. Chin Med J (Engl) 117: 213-218, 2004.

23. Xu HT, Wei Q, Liu Y, et al: Overexpression of axin downregulates TCF-4 and inhibits the development of lung cancer. Ann Surg Oncol 14: 3251-3259, 2007.

24. Cheung KK, Mok SC, Rezaie P and Chan WY: Dynamic expression of Dab2 in the mouse embryonic central nervous system. BMC Dev Biol 8: 76, 2008.

25. Martin JC, Herbert BS and Hocevar BA: Disabled-2 downregulation promotes epithelial-to-mesenchymal transition. $\mathrm{Br} \mathrm{J}$ Cancer 103: 1716-1723, 2010.

26. Bagadi SA, Prasad CP, Srivastava A, Prashad R, Gupta SD and Ralhan R: Frequent loss of Dab2 protein and infrequent promoter hypermethylation in breast cancer. Breast Cancer Res Treat 104: 277-286, 2007.

27. Xu HT, Yang LH, Li QC, Liu SL, Liu D, Xie XM and Wang EH: Disabled-2 and Axin are concurrently colocalized and underexpressed in lung cancers. Hum Pathol 42: 1491-1498, 2011.

28. Tong JH, Ng DC, Chau SL, et al: Putative tumour-suppressor gene DAB2 is frequently down regulated by promoter hypermethylation in nasopharyngeal carcinoma. BMC Cancer 10: 253, 2010.

29. Hannigan A, Smith P, Kalna G, et al: Epigenetic downregulation of human disabled homolog 2 switches TGF-beta from a tumor suppressor to a tumor promoter. J Clin Invest 120: 2842-2857, 2010

30. Yang Y, Zhang Q, Xu F, Chang C and Li X: Aberrant promoter methylation of Dab2 gene in myelodysplastic syndrome. Eur J Haematol 89: 469-477, 2012. 